\title{
Diagnostic performance of intraoperative assessment in grade 2 endometrioid endometrial carcinoma
}

\author{
Antonio Bandala-Jacques ${ }^{1,2}$, David Cantú-de-León ${ }^{3}$, Delia Pérez-Montiel ${ }^{4}$, Rosa A. Salcedo-Hernández \\ Diddier Prada ${ }^{1,6,7}$, Aarón González-Enciso ${ }^{8}$, Arely Gonzalez-Valdés ${ }^{5}$ and Salim Abraham Barquet-Muñoz ${ }^{5^{*}}$ (10
}

\begin{abstract}
Background: Endometrial carcinoma is the most common gynecologic malignancy in developed countries. Grade 2 carcinoma is associated with pelvic lymph-node metastasis, depending on selected risk factors. Intraoperative assessment (IOA) can identify patients at risk for lymph node metastasis who should undergo staging surgery. Our objective was to establish the diagnostic precision of IOA in determining the need for surgical staging in grade 2 endometrioid endometrial carcinoma.
\end{abstract}

Methods: Two hundred twenty-two patients underwent IOA. Results were compared to the final pathology report. The accuracy of the IOA parameters was calculated. Variables were evaluated in patients with positive versus negative IOA. Overall and disease-free survivals were calculated according to IOA, lymphadenectomy, and nodal metastasis.

Results: IOA was positive in 80 patients. It showed an accuracy of $76.13 \%$ when compared with the postoperative assessment. The best individual parameter was myometrial invasion. Nodal metastasis was observed in 16 patients in the positive IOA group and 7 patients in the negative group. Patients with lymph node metastasis had a 5-year overall survival rate of $80.9 \%$, whereas patients without metastasis had a 5 -year overall survival rate of $97.9 \%$.

Conclusions: IOA is an adequate tool to identify high-risk patients in grade 2 endometrial carcinoma. Myometrial invasion is the individual parameter that yields the highest diagnostic precision.

Keywords: Endometrial cancer, Endometrioid adenocarcinoma, Surgical diagnostic technique, Frozen sections, Lymphadenectomy, Myometrial invasion

\section{Background}

Endometrial carcinoma is the most common gynecologic malignancy in high- and middle-income countries [1]. It is the fourth most common cancer in women and has had a rising incidence trend in the last decade [2]. The most common histology is endometrioid, and its treatment consists of hysterectomy with bilateral salpingooophorectomy [3]. Patients may undergo pelvic and paraaortic lymphadenectomy depending on their risk for

\footnotetext{
*Correspondence: sbarquet@gmail.com

${ }^{5}$ Department of Gynecology, Instituto Nacional de Cancerología, Mexico City, Mexico

Full list of author information is available at the end of the article
}

lymph node metastasis. Lymph node metastasis is one of the most important prognostic factors for endometrial cancer, as it renders a tumor with a high risk for recurrence that calls for adjuvant therapy [4].

Lymph node metastasis cannot be determined without node dissection, especially if metastasis is microscopic, but it strongly associates with a high tumor grade and deep myometrial invasion, which can be determined intraoperatively, through intraoperative gross examination, frozen section biopsy, and/or touch imprint cytology [5]. If the intraoperative assessment (IOA) shows high-risk factors, complete surgical staging is recommended [6]. That is, as pelvic and paraaortic lymphadenectomy 
procedures increase morbidity and the surgical time, they are not performed routinely [7]. Furthermore, the final pathology report can differ from intraoperative findings, and IOAs could lead to unnecessary surgeries in women with low-risk disease [8].

According to the 2009 FIGO staging system, grade 2 endometrial carcinomas are defined as tumors with, from 6 to $50 \%$ of solid non-glandular, non-squamous growth architectural elements [9]. Grade 2 tumors are considered at low to medium risk for node metastasis, especially when combined with other factors, such as the depth of myometrial invasion and lymphovascular invasion $[10,11]$. For example, grade 2 endometrioid carcinomas with less than $50 \%$ myometrial invasion have a $4.8 \%$ probability of lymph node metastasis [12]. However, in the case of grade 2 endometrioid tumors that infiltrates more than $50 \%$ of the myometrium, the probability of pelvic lymph node metastasis increases to $15 \%[13]$.

Therefore, an accurate judgment of the elements in the IOA is important for deciding treatment strategies. The evaluation of multiple parameters together, such as grade and myometrial, cervical, or ovarian involvement, may prove accurate in discriminating high- from lowrisk patients for lymph node metastasis [14]. Patients with a high risk for lymph node metastasis should undergo lymphadenectomy, while those with a low risk can be spared the risks and complications of a staging surgery.

The purpose of this study was to establish the precision of intraoperative assessment in determining the need for surgical staging in grade 2 endometrioid endometrial carcinoma of the uterus.

\section{Materials and methods}

Data used in this study was obtained from electronic hospital records of patients treated at our institution between January 2016 and December 2018. The protocol was approved by our Institutional Review Board, with approval reference $\operatorname{Rev} / 020 / 20$. The inclusion criteria were women older than 18 years with grade 2 endometrioid endometrial carcinoma as confirmed by our institution's Pathology Department that underwent surgical intraoperative assessment. The exclusion criteria were incomplete data, non-endometrioid histology, or final pathology report of a histological grade other than two.

Patients were analyzed according to intraoperative assessment positivity. Intraoperative assessment consisted of gross examination and frozen section biopsy. A positive IOA was defined as myometrial invasion greater than $50 \%$ in depth or with cervical, serous, or ovarian involvement. Disease-free survival was defined as the time frame between surgical treatment and disease recurrence or the date last seen. Overall survival was defined as the time frame between diagnosis and death or the date last seen.

Patients underwent lymphadenectomy in accordance with both the IOA results and clinical criteria as determined by gynecologic oncologists, such as enlarged lymph nodes with abnormal consistency or conglomerated retroperitoneal lymph node masses. Pelvic lymphadenectomy consisted of the bilateral dissection of all lymph nodes between the circumflex vein as the inferior margin, $2 \mathrm{~cm}$ above the bifurcation of the external iliac artery as the superior margin, the genitocrural nerve as the lateral margin, the superior vesical artery as the medial margin, and the obturator nerve as the dissection floor. Paraaortic lymphadenectomy had ureters as the lateral margins, the infrarenal vein as the superior margin, and $2 \mathrm{~cm}$ above the external iliac artery bifurcation as the inferior margin.

\section{Statistical analysis}

For the descriptive analysis, the Shapiro-Wilk test was used to identify the normality of the continuous variables. The mean and standard deviation (SD) were used for continuous normal variables, and the median and interquartile range (IQR) were used for continuous nonnormal variables. Absolute and relative frequencies were used for categorical variables. For the comparative analysis, Student's $t$ test, Wilcoxon's rank-sum test, the chisquared test, or Fisher's exact test were used depending on the analyzed variable. Survival curves were generated with Kaplan-Meier estimator and compared with the log-rank test. Logistic regression was used to obtain the odds ratios (ORs) and establish factors associated with frozen section biopsies. Diagnostic tests with the area under the curve (AUC), sensitivity, specificity, and predictive values were performed to estimate the diagnostic value of intraoperative frozen section biopsy, taking the definitive pathology report as the reference standard. Statistical significance was defined as a $p$ value $<0.05$. Statistical analysis was performed with the STATA software, version 13.0 (College Station, TX, licensed to the author).

\section{Results}

A total of 222 patients met the inclusion criteria and were analyzed. The mean patient age was 54.5 years (SD 11.7). The median body mass index was 31 (IQR 27.135.9) $\mathrm{kg} / \mathrm{m}^{2}$. Postoperative stages were I in $162(73 \%) \mathrm{pa}-$ tients, II in $25(11.2 \%)$, III in $30(13.5 \%)$, and IV in 5 (2.2\%). Eighty-seven (39.2\%) patients underwent lymphadenectomy.

All patients had an IOA. Frozen section biopsy was positive in $80(36 \%)$ patients. The IOA was positive for ovarian involvement in $2(0.9 \%)$ patients, uterine serosa involvement in 12 (5.4\%), and cervical involvement in 29 
(13\%). Myometrial invasion was superficial in $34(15.3 \%)$ patients, less than 50\% deep in 137 (61.7\%), and greater than $50 \%$ deep in $51(23 \%)$. The presurgical tumor grade was grade 1 in $7(11.1 \%)$ patients, grade $2(73 \%)$ in 46 patients, and grade 3 in $9(14.3 \%)$ patients. In the final pathology report, the median tumor size was $40 \mathrm{~mm}$ (IQR 30-35), there was ovarian involvement in $10(4.5 \%)$ patients, uterine serosa involvement in $3(1.4 \%)$, and cervical involvement in 55 (24.8\%). Myometrial invasion was superficial in 34 (15.3\%) patients, less than 50\% gross depth invasion in 133 (59.5\%), and greater than $50 \%$ gross depth invasion in $55(24.8 \%)$. There was lymph node metastasis in $23(10.4 \%)$ patients (Table 1$)$.

The IOA showed an accuracy of $76.1 \%$ when compared with the postoperative assessment. It had an AUC of 0.74 (95\% confidence interval [CI] 0.68-0.8), a sensitivity of $65.5 \%$, a specificity of $83 \%$, a positive predictive value of $71.3 \%$, and a negative predictive value of $78.9 \%$. Myometrial invasion had an AUC of 0.76 (95\% CI 0.69-

Table 1 Tumor characteristics as reported in the intraoperative assessment and final pathology report, $n=222$

\begin{tabular}{|c|c|}
\hline \multicolumn{2}{|l|}{ Intraoperative assessment report } \\
\hline Positive intraoperative assessment & $80(36 \%)$ \\
\hline \multicolumn{2}{|l|}{ Myometrial invasion } \\
\hline Superficial & $34(15.3 \%)$ \\
\hline$\leq 50 \%$ & $137(61.7 \%)$ \\
\hline$>50 \%$ & $51(23 \%)$ \\
\hline Cervical involvement & $29(13 \%)$ \\
\hline Uterine serosa involvement & $12(5.4 \%)$ \\
\hline Ovarian involvement & $2(0.9 \%)$ \\
\hline \multicolumn{2}{|l|}{ Grade, $n=63(28.37 \%)$} \\
\hline 1 & $7(11.1 \%)$ \\
\hline 2 & 47 (74.6\%) \\
\hline 3 & $9(14.3 \%)$ \\
\hline \multicolumn{2}{|l|}{ Final pathology report } \\
\hline Tumor size, mm & $40(30-35)^{a}$ \\
\hline \multicolumn{2}{|l|}{ Myometrial invasion } \\
\hline Superficial & $34(15.3 \%)$ \\
\hline$\leq 50 \%$ & $133(59.9 \%)$ \\
\hline$>50 \%$ & $55(24.8 \%)$ \\
\hline Uterine serosa involvement & $3(1.4 \%)$ \\
\hline Ovarian involvement & $10(4.5 \%)$ \\
\hline Cervical involvement & $55(24.8 \%)$ \\
\hline Lymphovascular permeation & $41(18.5 \%)$ \\
\hline \multicolumn{2}{|l|}{ Lymph node metastasis $(n=89)$} \\
\hline Yes & $23(10.4 \%)$ \\
\hline No & $66(29.7 \%)$ \\
\hline
\end{tabular}

${ }^{a}$ Median (interquartile range)
0.83 ) and an accuracy of $82.4 \%$ when compared with myometrial invasion in the final pathology report. Cervical involvement had an AUC of 0.61 (95\% CI 0.550.68 ) and an accuracy of $77.5 \%$. Uterine serosa involvement had an AUC of 0.47 (95\% CI 0.46-0.49) and an accuracy of $93.2 \%$. Ovarian involvement had an AUC of 0.55 (95\% CI $0.45-0.65)$ and an accuracy of $95.5 \%$. The rate of lymph node metastasis according to each parameter's positivity was $25.5 \%$ for myometrium, $13.8 \%$ for cervix, $33.3 \%$ for uterine serosa, and $50 \%$ for ovary (Table 2).

A total of $80(36 \%)$ patients had positive IOA, and 142 (64\%) were negative. Lymphadenectomy was performed on $69(86.3 \%)$ patients in the positive IOA group and 18 $(12.7 \%)$ in the negative IOA group $(p<0.001)$. There was lymph node metastasis in $16(20 \%)$ patients in the positive IOA group and 7 (4.9\%) patients in the negative IOA group $(p<0.001)$. Regarding intraoperative complications, patients in the positive IOA group had more intraoperative bleeding (375 ml, IQR 80-300) than those in the negative IOA group (150 ml, IQR 200-550) $(p<$ $0.001)$. Likewise, there were $8(10 \%)$ blood transfusions in the positive frozen section biopsy group and none $(0 \%)$ in the negative frozen section biopsy group $(p<$ 0.001). There were no differences found regarding age, menarche, menopause, weight, reintervention, stage IV disease, or ICU admission (Table 3).

The median follow-up duration was 43.8 (IQR 24.47$65.8)$ months. The 5 -year overall survival rate for all patients was $95.3 \%$ (95\% CI 89.5-97.9). Patients with a positive IOA had a 5 -year overall survival rate of $92 \%$ (95\% CI 79.05-97.1), whereas patients with a negative IOA had a 5 -year overall survival rate of $97.7 \%$ (95\% CI 93.08-99.26) $(p=0.257)$. Patients who underwent lymphadenectomy had a 5-year overall survival rate of 94.7\% (95\% CI 83.4-98.4), whereas those who did not undergo lymphadenectomy had a 5-year overall survival rate of 96.2\% (95\% CI 89.8-98.6) ( $p=0.99$ ). Patients with lymph node metastasis had a 5-year overall survival rate of $80.9 \%$ (95\% CI 65.3-96.5), whereas patients without lymph node metastasis had a 5-year overall survival rate of 97.9\% (95\% CI 86.4-99.7) ( $p=0.04$ ) (Fig. 1).

The 5 -year disease-free survival rate was $91.3 \%$ for all patients. Patients with a positive IOA had a 5-year disease-free survival rate of $86 \%$ (95\% CI 73.5-92.91), whereas patients with a negative IOA had a 5-year disease-free survival rate of $94.4 \%$ (95\% CI 87.29-97.62) $(p=0.177)$. Patients who underwent lymphadenectomy had a 5-year disease-free survival rate of $91.1 \%$ (95\% CI 80.94-96), whereas patients who did not undergo lymphadenectomy had a 5-year disease-free survival rate of 91.3\% (95\% CI 82.4-95.9) ( $p=0.789)$. Patients with lymph node disease had a 5-year disease-free survival rate of $91.3 \%$ (95\% CI 82.4-95.8), whereas patients 
Table 2 Diagnostic accuracy of intraoperative assessment parameters

\begin{tabular}{|c|c|c|c|c|c|c|c|c|c|c|}
\hline & $N(\%)$ & LN metastasis rate (\%) & AUC (95\% CI) & Sen & Spe & PPV & NPV & LR+ & LR- & Accuracy \\
\hline Overall IOA + & $80(36)$ & $16(20)$ & $0.74(0.68-0.80)$ & 65.5 & 83 & 71.3 & 78.9 & 3.9 & 0.42 & 76.1 \\
\hline Myometrium IOA+ & $55(24.8)$ & $14(25.5)$ & $0.76(0.69-0.83)$ & 64.3 & 88.6 & 65.4 & 88 & 5.6 & 0.4 & 82.4 \\
\hline Cervix IOA + & $29(13)$ & $4(13.8)$ & $0.61(0.55-0.68)$ & 30.9 & 92.8 & 58.6 & 80.3 & 4.3 & 0.74 & 77.5 \\
\hline Serous IOA + & $12(5.4)$ & $4(33.3)$ & $0.47(0.46-0.49)$ & 0 & 94.5 & 0 & 98.6 & 0 & 1.5 & 93.2 \\
\hline Ovarian $\mathrm{IOA}+$ & $2(0.9)$ & $1(50)$ & $0.55(0.45-0.65)$ & 10 & 99.5 & 50 & 95.9 & 21.2 & 0.9 & 96 \\
\hline
\end{tabular}

AUC area under the curve, Sen sensitivity, Spe specificity, PPV positive predictive value, NPV negative predictive value, $L R$ likelihood ratio, IOA intraoperative assessment

without lymph node disease had a 5-year disease-free survival rate of $93.9 \%$ (95\% CI 81.6-98) (Fig. 2).

\section{Discussion}

The main findings of our study were that in patients with grade 2 endometrioid endometrial carcinomas, IOA is an adequate tool to determine the need for lymph node evaluation. Out of the IOA parameters, myometrial invasion had the greatest diagnostic precision. Furthermore, undergoing the procedure does not impact overall survival or disease-free survival.

Table 3 Comparative analysis according to the intraoperative assessment results, $n=222$

\begin{tabular}{|c|c|c|c|}
\hline & $\begin{array}{l}\text { IOA negative } \\
142(64 \%)\end{array}$ & $\begin{array}{l}\text { IOA positive } \\
80(36 \%)\end{array}$ & $P$ \\
\hline$\overline{\mathrm{Age}^{\mathrm{a}}}$ & $53.7 \pm 11.4$ & $56 \pm 12.2$ & 0.166 \\
\hline Menopause & $108(76 \%)$ & $62(77.5 \%)$ & 0.807 \\
\hline Weight, kg ${ }^{\mathrm{b}}$ & $74.5(65.5-85.5)$ & $68.5(60-80.5)$ & 0.079 \\
\hline$B M l^{\mathrm{a}}$ & $32.6 \pm 6.7$ & $31.1 \pm 7.1$ & 0.131 \\
\hline \multicolumn{4}{|l|}{ Surgical stage } \\
\hline I & $120(84.5 \%)$ & $42(52.5 \%)$ & $<0.001$ \\
\hline$\|$ & $12(8.5 \%)$ & $13(16.3 \%)$ & \\
\hline III & $8(5.6 \%)$ & $22(27.5 \%)$ & \\
\hline IV & $2(1.4 \%)$ & $3(3.8 \%)$ & \\
\hline Lymph node metastasis & $7(4.9 \%)$ & $16(20 \%)$ & $<0.001$ \\
\hline Lymphadenectomy & $18(12.7 \%)$ & $69(86.3 \%)$ & $<0.001$ \\
\hline Bleeding, $\mathrm{ml}^{\mathrm{b}}$ & $150(80-300)$ & $375(200-550)$ & $<0.001$ \\
\hline Transfusion & $0(0 \%)$ & $8(10 \%)$ & $<0.001$ \\
\hline Reintervention & $2(1.4 \%)$ & $3(3.8 \%)$ & 0.277 \\
\hline $\mathrm{ICU}$ & $2(1.4 \%)$ & $5(6.3 \%)$ & 0.07 \\
\hline Adjuvant therapy & $44(31 \%)$ & $62(77.5 \%)$ & $<0.001$ \\
\hline Radiotherapy & 41 (28.9\%) & $58(72.5 \%)$ & 0.94 \\
\hline Chemotherapy & $8(5.6 \%)$ & $22(27.5 \%)$ & 0.049 \\
\hline Recurrence of disease & $7(4.9 \%)$ & $8(10 \%)$ & 0.156 \\
\hline
\end{tabular}

FSB frozen section biopsy, $B M I$ body mass index, ICU intensive care unit, $I O A$ intraoperative assessment

a Mean \pm standard deviation

${ }^{\mathrm{b}}$ Median (interquartile range)
The usefulness of an IOA is debatable, as its misinterpretation can lead to unnecessary surgeries [15]. In our study, the IOA had a $76 \%$ concordance rate with the final pathology report, with the best overall predictor being myometrial invasion. Studies have found myometrial invasion to be a good predictor of node metastasis, with a sensitivity as high as $86 \%$ [7]. Our overall accuracy for myometrial invasion was $82 \%$. While cervical involvement had a precision of $77 \%$, it had a low sensitivity (30\%). This might be because the IOA is not able to detect microscopic cervical foci, which can be detected only in the final postoperative assessment [16]. Although there is great variation in the reported concordance of the IOA with the final pathology report between institutions, an analysis of both by a trained gynecologic pathologist improves the accuracy of the IOA [17].

Incomplete surgical staging can have consequences such as extended-field radiotherapy or further staging procedures if the final pathology report demonstrates high risk [18]. Grade determination by curettage alone is unreliable, as it can differ from the final report in over $50 \%$ of cases, and other techniques such as magnetic resonance imaging are not always available in all centers. A gross evaluation of the uterus improves this accuracy [19]. A study found a concordance rate of $86 \%$ between frozen sections and paraffin sections for grade 2 tumors, which was lower than those for grade 1 or 3 tumors. The study also found an underestimation of $8.2 \%$ [20]. Our institution does not always include tumor grade in the presurgical report, but of those available, there is $75 \%$ concordance and $11 \%$ underestimation.

After IOA has identified patients at risk for lymph node metastasis, they can undergo lymphadenectomy. Lymphadenectomy plays only a diagnostic role, does not impact the prognosis, and does not increase the risk of intraoperative complications [21]. Our study confirmed that pelvic and paraaortic lymphadenectomy in grade 2 endometrioid endometrial carcinoma had no impact on overall survival or disease-free survival and was not associated with a higher risk of intraoperative complications. However, lymphadenectomy did help identify patients with lymph node disease, which does impact survival [22]. Lymph node metastasis significantly affects disease 

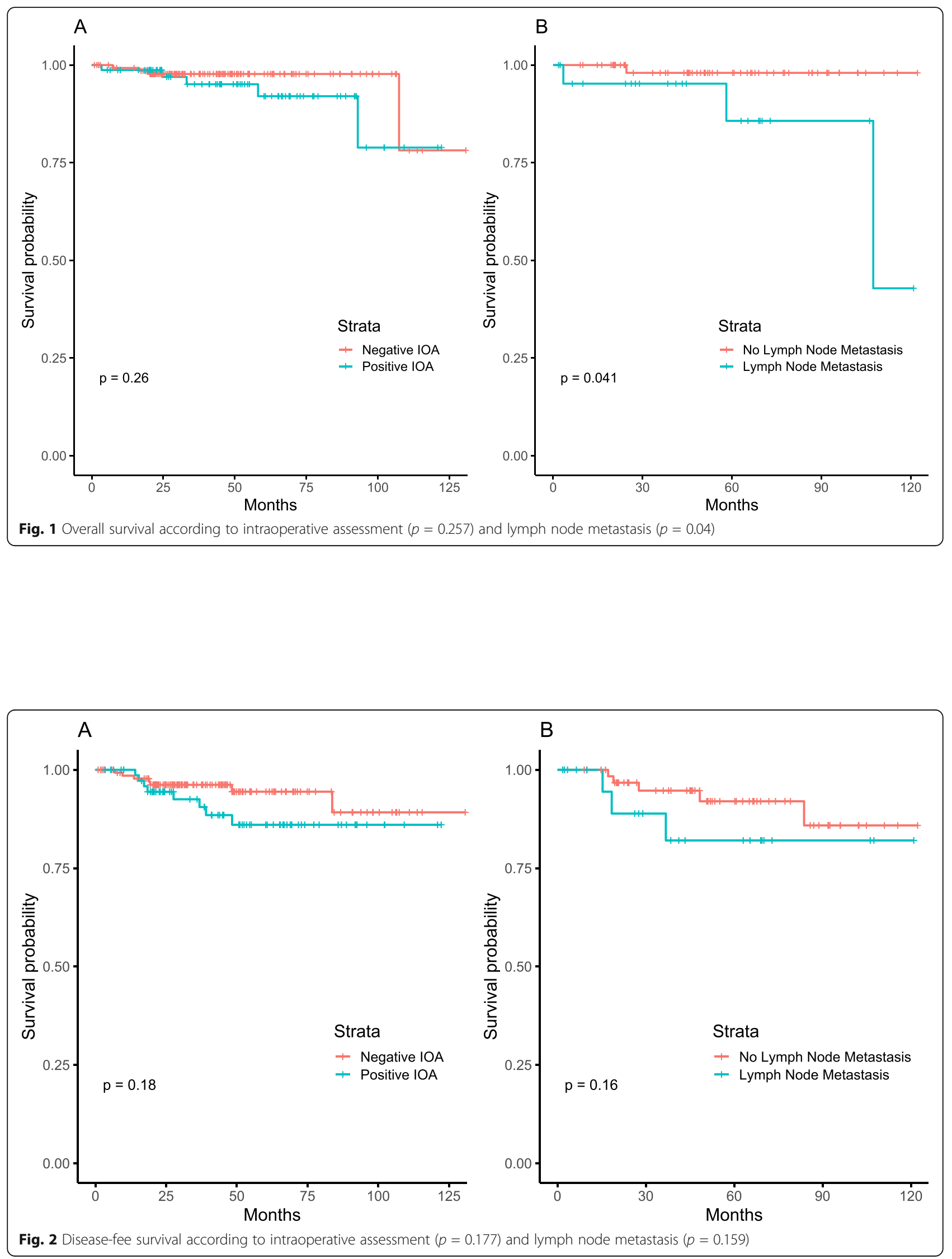
prognosis and can reduce 5-year overall survival from as high as $91 \%$ to as low as $44 \%$ [23]. In our study, having positive lymph nodes reduced the 5-year overall survival rate from 97.9 to $85.7 \%$. A different study found a reduction in the 5-year overall survival rate from 96.5 to $77.6 \%$ in the presence of pelvic node metastasis and worse outcomes with paraaortic node metastasis [24]. As this is an important prognostic factor, it is critical to identify patients with lymph node metastasis. Even with novel techniques, the use of IOA with gross examination and frozen section is widespread and can help determine high-risk factors that warrant surgical staging with lymphadenectomy. On the other hand, sometimes patients with negative frozen section biopsies undergo lymphadenectomy if there is high clinical suspicion of node metastasis. In our study, 18 out of the 142 patients with a negative frozen section received lymphadenectomy, out of which 7 (38.9\%) were positive for node metastasis. If clinical suspicion remains high after a negative IOA, patients should undergo lymphadenectomy regardless.

Sentinel lymph node mapping has become an increasingly popular alternative for lymphadenectomy due to its shorter operative time and reduced number of lymphatic complications [25]. Although it is now also included as a standard technique for nodal evaluation, large cohorts have found that its survival rate is similar to that of systematic lymphadenectomy [26]. As sentinel lymph node biopsy is not yet widely available in low-income countries, it is safe to continue comprehensive lymphadenectomy with frozen sections if available.

The limitations of our study include its retrospective nature. Furthermore, our institution does not report tumor grade on most IOAs, so we were unable to evaluate tumor grade mismatch in most patients. The main strength of our study is that it provides insights of the performance of a widespread diagnostic technique when applied exclusively to grade 2 tumors, and that it was performed in an oncologic institution where specialized gynecologic pathologists review all specimens.

\section{Conclusions}

In conclusion, our study supports the use of IOA with gross examination and frozen section biopsy for determining the need for lymph node evaluation by lymphadenectomy in patients with grade 2 endometrioid endometrial carcinomas. Within the IOA, myometrial invasion yields the highest overall diagnostic accuracy. Patients with negative frozen sections should undergo lymphadenectomy regardless if clinical suspicion remains high. Lymphadenectomy does not affect diseasefree survival or overall survival, but it does help to identify patients with node metastasis.

\section{Abbreviations}

IOA: Intraoperative assessment; AUC: Area-under-curve

\section{Acknowledgements}

Not applicable.

\section{Authors' contributions}

SABM and AGV conceived the idea and designed the work. DCDL, AGE, RSH, and DPM obtained the data. SABM and DP analyzed the data. ABJ interpreted the data. $A B J$ wrote the manuscript. DCDL critically revised the manuscript. All authors read and approved the final manuscript. All authors are accountable for the contents of this work.

\section{Funding}

There was no funding for this study.

\section{Availability of data and materials}

The datasets generated and/or analyzed during the current study are not publicly available due to hospital policy but are available from the corresponding author on reasonable request.

Ethics approval and consent to participate

This study was approved by our Institutional Review Board, with reference Rev/020/20.

\section{Consent for publication}

Not applicable.

\section{Competing interests}

The authors declare that they have no competing interests.

\section{Author details}

${ }^{1}$ Biomedical Cancer Research Unit, Instituto Nacional de Cancerología, Mexico City, Mexico. ${ }^{2}$ Biomedical Investigations Institute, Universidad Nacional Autonóma de México, Mexico City, Mexico. ${ }^{3}$ Instituto Nacional de Cancerología, Mexico City, Mexico. ${ }^{4}$ Department of Pathology, Instituto Nacional de Cancerología, Mexico City, Mexico. ${ }^{5}$ Department of Gynecology, Instituto Nacional de Cancerología, Mexico City, Mexico. ${ }^{6}$ Department of Biomedical Informatics, Faculty of Medicine, Universidad Nacional Autónoma de México, Mexico City, Mexico. ${ }^{7}$ Department of Environmental Health Sciences, Mailman School of Public Health, Columbia University, New York City, USA. ${ }^{8}$ Department of Surgery, Instituto Nacional de Cancerología, Mexico City, Mexico

Received: 8 September 2020 Accepted: 19 October 2020

Published online: 30 October 2020

\section{References}

1. Piulats JM, Guerra E, Gil-Martín M, Roman-Canal B, Gatius S, Sanz-Pamplona $R$, et al. Molecular approaches for classifying endometrial carcinoma. Gynecol Oncol. 2017;145(1):200-7.

2. Ali AT. Risk factors for endometrial cancer. Ceska Gynekol. 2013;78(5):448-59.

3. Sorosky JI. Endometrial cancer. Obstet Gynecol. 2012;120(2 Pt 1):383-97.

4. Wright JD, Medel NIB, Sehouli J, Fujiwara K, Herzog TJ. Contemporary management of endometrial cancer. Lancet. 2012;379(9823):1352-60.

5. Freeman SJ, Aly AM, Kataoka MY, Addley HC, Reinhold C, Sala E. The revised FIGO staging system for uterine malignancies: implications for MR imaging. Radiographics. 2012;32(6):1805-27.

6. Sala P, Moran E, Paolucci R, Menoni S, Fulcheri E, Costantini S, et al. Selection of patients with high risk endometrial cancer for surgical staging according to the evaluation of pre- and intraoperative risk factors. Minerva Ginecol. 2016;68(1):21-8.

7. Acikalin A, Gumurdulu D, Bagir EK, Torun G, Guzel AB, Zeren H, et al. The guidance of intraoperative frozen section for staging surgery in endometrial carcinoma: frozen section in endometrial carcinoma. Pathol Oncol Res. 2015;21(1):119-22.

8. Frumovitz M, Slomovitz BM, Singh DK, Broaddus RR, Abrams J, Sun CC, et al. Frozen section analyses as predictors of lymphatic spread in patients with early-stage uterine cancer. J Am Coll Surg. 2004;199(3):388-93.

9. Maheshwari A, Gupta S, Prat J. A proposal for updating the staging of endometrial cancer. Int J Gynecol Obstet. 2019;145(2):245-52. 
10. Güngördük K, Firat Cüylan Z, Kahramanoglu I, Oge T, Akbayir O, Dede M, et al. Risk factors for recurrence in low-risk endometrial cancer: a casecontrol study. Oncol Res Treat. 2018:41:466-70.

11. Parkash V, Fadare O. Endometrial carcinoma: grossing, frozen section evaluation, staging, and sentinel lymph node evaluation. Surg Pathol Clin. 2019;12(2):329-42.

12. Wu Y, Zhu H, Sun J, Wang $X$. Accuracy of frozen section in management and prediction of lymph node metastasis in endometrial carcinoma. Gynecol Minim Invasive Ther. 2015;4:126-31.

13. Rathod PS, Shakuntala PN, Pallavi VR, Kundaragi R, Shankaranand B, Vijay CR, et al. The risk and pattern of pelvic and para aortic lymph nodal metastasis in patients with intermediate and high risk endometrial cancer. Indian J Surg Oncol. 2014;5(2):109-14

14. Karabagli P, Ugras S, Yilmaz BS, Celik C. The evaluation of reliability and contribution of frozen section pathology to staging endometrioid adenocarcinomas. Arch Gynecol Obstet. 2015;292(2):391-7.

15. Senol T, Polat M, Özkaya E, Karateke A. Misinterpretation of frozen section in endometrial cancer cases: does it have any effect on disease-free and overall survival? Int J Gynecol Pathol. 2017;36(6):550-4.

16. Santoro A, Piermattei A, Inzani F, Angelico G, Valente M, Arciuolo D, et al. Frozen section accurately allows pathological characterization of endometrial cancer in patients with a preoperative ambiguous or inconclusive diagnoses: our experience. BMC Cancer. 2019;19:1096.

17. Wang X, Li L, Cragun JM, Chambers SK, Hatch KD, Zheng W. Assessment of the role of intraoperative frozen section in guiding surgical staging for endometrial cancer. Int J Gynecol Cancer. 2016;26(5):918-23.

18. Kumar S, Bandyopadhyay S, Semaan A, Shah JP, Mahdi H, Morris R, et al. The role of frozen section in surgical staging of low risk endometrial cancer PLoS One. 2011;6(9):e21912.

19. Noumoff JS, Menzin A, Mikuta J, Lusk EJ, Morgan M, LiVolsi VA. The ability to evaluate prognostic variables on frozen section in hysterectomies performed for endometrial carcinoma. Gynecol Oncol. 1991;42(3):202-8.

20. Turan T, Oguz E, Unlubilgin E, Tulunay G, Boran N, Demir OF, et al. Accuracy of frozen-section examination for myometrial invasion and grade in endometrial cancer. Eur J Obstet Gynecol Reprod Biol. 2013;167(1):90-5.

21. Guo W, Cai J, Li M, Wang H, Shen Y. Survival benefits of pelvic lymphadenectomy versus pelvic and para-aortic lymphadenectomy in patients with endometrial cancer. Med. 2018;97(1):e9520.

22. Greco P, Martinello R, Indraccolo U, Borghi C, Bonaccorsi G, Scutiero G. Outcome of endometrial cancer after lymphadenectomy: a single cente retrospective analysis with long-lasting follow-up. Minerva Ginecol. 2017; 69(5):405-12.

23. Uharček P. Prognostic factors in endometrial carcinoma. J Obstet Gynaeco Res. 2008;34(5):776-83.

24. Todo Y, Takeshita S, Okamoto K, Yamashiro K, Kato H. Implications of paraaortic lymph node metastasis in patients with endometrial cancer without pelvic lymph node metastasis. J Gynecol Oncol. 2017;28(5):e59.

25. Geppert B, Lönnerfors C, Bollino M, Persson J. Sentinel lymph node biopsy in endometrial cancer-feasibility, safety and lymphatic complications. Gynecol Oncol. 2018;148(3):491-8.

26. Rossi EC. Current state of sentinel lymph nodes for women with endometrial cancer. Int J Gynecol Cancer. 2019;3(40):613-21.

\section{Publisher's Note}

Springer Nature remains neutral with regard to jurisdictional claims in published maps and institutional affiliations.

Ready to submit your research? Choose BMC and benefit from:

- fast, convenient online submission

- thorough peer review by experienced researchers in your field

- rapid publication on acceptance

- support for research data, including large and complex data types

- gold Open Access which fosters wider collaboration and increased citations

- maximum visibility for your research: over $100 \mathrm{M}$ website views per year

At $\mathrm{BMC}$, research is always in progress.

Learn more biomedcentral.com/submissions 